\title{
Conjugate Heat Transfer in a Turbine Vane and Thermal Barrier Coating
}

\author{
Rami Salem, Marc Errera, Julien Marty \\ ONERA- The French Aerospace Lab, Aerodynamics - Aeroelastics - Aeroacoustics Department \\ 8 Rue des Vertugadins, 92190 Meudon, France \\ rami.salem@onera.fr; marc.errera@onera.fr; julien.marty@onera.fr
}

\begin{abstract}
The high pressure (HP) turbine is subject to high temperature inlet flow non-uniformities resulting from the combustion chamber. This paper presents a new fluid - solid interface treatment for conjugate heat transfer (CHT) applied to two configurations of interest in the design of high pressure turbine blades to accurately estimate the temperature in the blade. The computational fluid dynamics (CFD) solver elsA, and a solid conduction solver, Zset, both developed at the French Aerospace Lab (ONERA), have been used for all simulations. Two problems, typical of a high pressure turbine, are studied: (1) a turbine vane with a hot-streak to present the validity of the coupling process during the design phase of a blade turbine. Results will be compared to CFD only simulation and experimental data. (2) A conjugate heat transfer simulation with a thermal barrier coating (TBC) on the solid side has also been studied. The TBC is known to better withstand high temperature but taking it into account in a CHT simulation is really difficult because of the strong fluid-solid interaction. This two configurations have really different characteristics in a coupled problem based on the Biot number, and the coupled methodology presented attempt to solve both of them using a Dirichlet-Robin interface condition.
\end{abstract}

Keywords: Turbine Vane, Aerothermal Simulation, Conjugates Heat Transfer, Thermal Barrier Coating.

\section{Introduction}

The continuous effort to increase aircraft engines performances has lead to the increase of the inlet temperature of the HP turbine. Such a high temperature combined with the inlet non-uniformities challenges the design of modern HP turbine blades. Determination of the heat loads such as wall temperature and heat fluxes is a key issue in modern gas turbine design. For more accurate prediction, having a reliable tool for fluid-solid wall treatment to reduce the uncertainties on the temperature seen by a HP turbine blade, is essential for turbine blades life-time at such severe operating conditions. Indeed, the advancement of turbine cooling systems has allowed engine design to exceed normal material temperature, but it has introduced complexities that have accentuated the thermal issues analysis greatly [1]. In high pressure turbines, it is common to find a thermal barrier coating (TBC) applied to the airfoil and the end wall surfaces. Usually the TBC is made of ceramic material. Ceramics are known to be more efficient in such environment with a low thermal conductivity compared to the alloys used in turbine blades. The currently state of the art TBC of a civil aircraft engine, have a thermal conductivity between 0.8 and $1.8 \mathrm{~W} / \mathrm{m} / \mathrm{k}$ and a thickness from 2 to $3 \mathrm{~mm}$ [2]. Thus, meshing such thin material makes the computation more CPU time consuming.

For both applications, the key element to consider for coupled methodology is the Biot number presented in [3]. The Biot number compares fluid thermal properties to the solid thermal properties. As there are presented, in this paper the turbine blade considered has a low Biot number while taking account the TBC increases considerably the Biot number.

Coupled procedures are the most appropriate way to interactively evaluate the boundary conditions and its thermal state. The coupling procedure includes a Dirichlet-Robin interface condition using a conventional serial staggered (CSS) algorithm, that have been tested previously [4][5][6], but this paper aims particularly to test the optimal coefficient in more complex aerothermal situations like hot-streak and thermal barrier coating. The optimal coupling coefficient has been highlighted in a theoretical study based on a normal mode analysis [3].

Results will be discussed and compared with results from CFD only simulation and conjugate heat transfer (CHT) simulation using a new coupling methodology. 


\section{Solvers and Coupling Strategy}

All the results presented are computed using the CFD elsA solver [7] on the fluid side, Zset [8] solver on the solid and CWIPI [9], a data exchanger library. The coupling scheme used is based on CSS methodology with DirichletRobin at the coupled interface using the optimal coefficient described in [3].

\subsection{Solver and Coupling Library}

The elsA software is used to solve the 3D-Reynolds averaged Navier-Stokes (RANS) equation with a finite volume method based on a multi-domain approach on a structured, unstructured or hybrid grids. Cell-centered space discretization scheme of Jameson with artificial viscosity is provided by elsA. There are also other classical numerical tools such as a second order Roe scheme for transport equation of turbulence models. To perform a steady simulation, time can be solved by an implicit lower upper scalar relaxation phase associated to a backward Euler scheme. A local time stepping is also available to reduce central processing unit (CPU) time. Computations presented for the turbine blade were performed with a 2 transport equations turbulence model, Menter's k- $\omega$, whereas computations performed on the flat plate with thermal barrier coating were performed with a 1 transport equation turbulence model, SpalartAllmaras.

Zset is a finite-element solver for mechanical design. In this paper Zset is simply used as a conduction solver. Since only the steady state is sought, the thermal equation can be reduced to a second order ordinary differential equation with appropriate thermal boundary conditions.

CWIPI aims to provide a fully parallel communication layer for mesh based coupling between several codes through MPI communications. The library takes into account all types of geometrical elements (polygon, polyhedral) with an unstructured description. CWIPI provides functionalities such as the construction of the communication graph between distributed geometric interfaces, interpolation of non-coincident meshes and exchange of coupled fields.

\subsection{Coupling Scheme}

In this paper, the coupling strategy consists in using a Dirichlet condition on the fluid side and a Robin condition on the solid side. This coupling procedure is referred to as the Dirichlet-Robin interface condition in the context of a steady CHT simulation. In this paper, a loosely-coupled CSS algorithm is used [10][11]. This method, presented in Fig. 1, consists in transferring data at a coupling time step corresponding to the slowest evolving domain (solver A). A linear interpolation is made by the slowest domain at each time step to estimate the variation of the other domain. The stability of this algorithm depends on that linear assumption but the solution is not necessarily badly estimated.

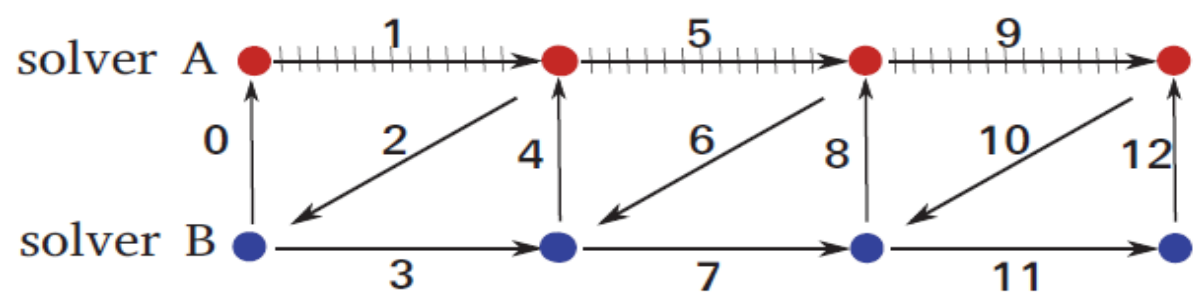

Fig. 1: Conventional serial staggered (CSS) algorithm.

The Dirichlet-Robin interface treatment used is defined as follow:

$$
\begin{gathered}
T_{f}=T_{s} \\
q_{s}+\alpha_{f} T_{s}=-q_{f}+\alpha_{f} T_{f}
\end{gathered}
$$


Where $T_{f}$ is the fluid temperature, $T_{s}$ the solid temperature, $q_{s}$ and $q_{f}$ are the solid normal heat flux and the fluid normal heat flux respectively. In this procedure, the $\alpha_{f}$ in the second equation plays a major role on stability. Indeed, lower lower is $\alpha_{f}$, faster the CHT simulation converges, but instabilities might appear. Thus, it is important to determine the proper value of this parameter for each cell coupled. The optimal $\alpha_{f}$ value was defined by Errera and Duchaine [6]:

$$
\alpha_{f}^{(o p t)}=\frac{K_{f}}{2}\left(1-\bar{D}_{f}\right)
$$

Where $K_{f}$ is the fluid conductance and $\bar{D}_{f}$ is a normalized Fourier number.

\section{Applications}

\subsection{Turbine Vane}

As it can be shown in Fig. 2, the HP blade turbine has to deal with strong non uniformities coming from the combustion chamber.
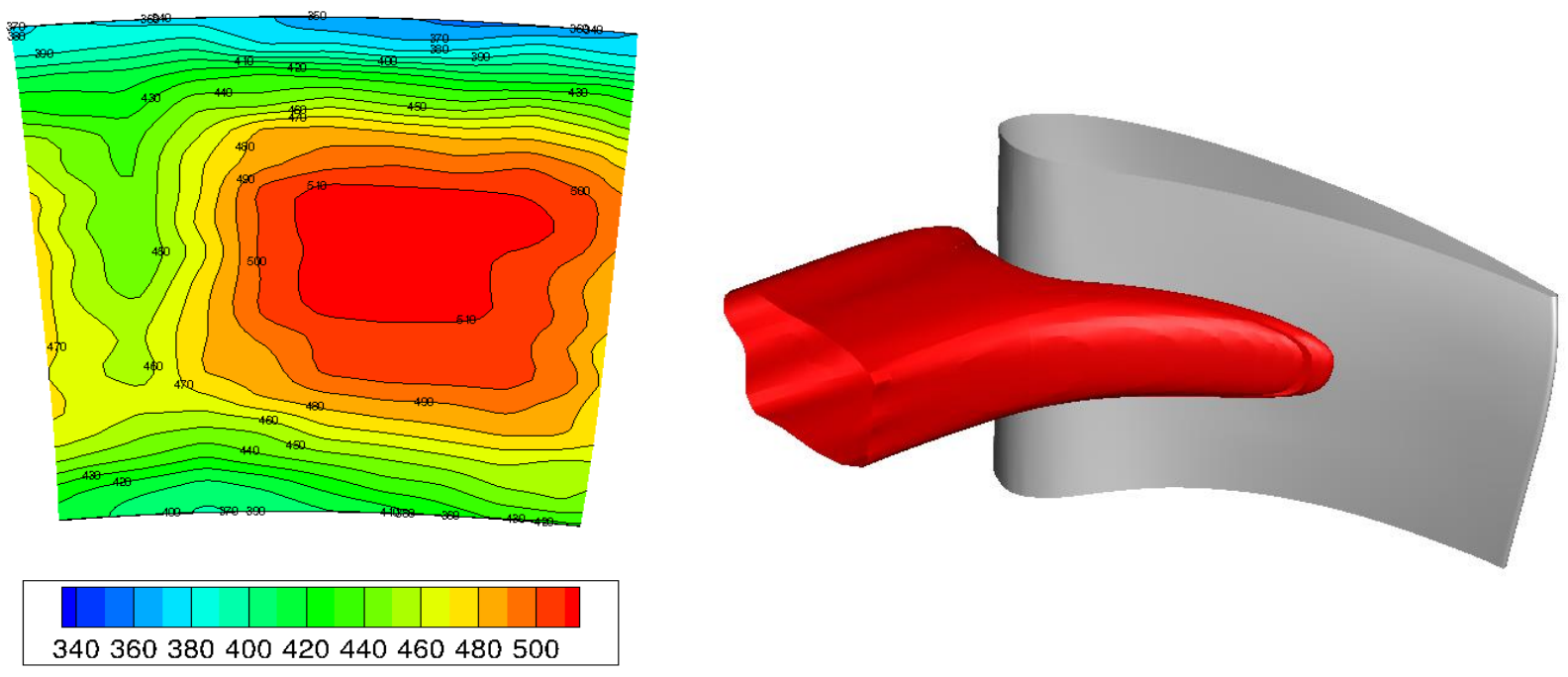

Fig. 2: Inlet temperature condition (left) and convection of hot fluid through NGV (right).

\section{Test Case Parameters}

The inlet mean temperature is $444 \mathrm{~K}$ and the wall temperature is $288 \mathrm{~K}$. For comparative study, the solid thermal conductivity imposed is the same as imposed by Montomoli et al. [12] and equal to $\lambda_{s}=12.2 \mathrm{~W} / \mathrm{m} / \mathrm{K}$. The Biot number introduced by Errera [3], Bi $=K_{f} / K_{s}$ defined by the ratio between the fluid conductance $K_{f}=\lambda_{f} / \Delta_{f}$ ( $\Delta_{f}$ is the fluid wall cell size) and solid conductance $K_{s}=\lambda_{s} / \Lambda$ ( $\Lambda$ is the solid wall thickness), gives an estimation of the fluid-solid interaction to avoid instabilities due to the coupling. The Biot number in this case is relatively low. A Robin condition is imposed on the solid side, then a local parameter is used to estimate the relaxation coefficient $\alpha_{f}$ that need to be transmitted to the solid. Since the thermal load at the blade wall is strongly non uniform, the local coefficient allows a better estimation of heat flux sent to the solid leading to a more stabilized CHT simulation. As the flow is subsonic, the static pressure is imposed using a radial equilibrium on the outflow boundary. This configuration is initially composed of 32 stators, since only one is considered here, periodic conditions are imposed for the flow outside interface boundaries. On the solid side, all non coupled boundaries are adiabatic. 


\section{Results}

Fig. 3 depicts the isentropic Mach on the nozzle guide vane (NGV) wall to have an aerodynamic validation of this methodology and the Nusselt numbers are compared to estimate the fluid-solid heat transfer at the blade wall.

The comparison is made with a CFD simulation at 50\% span. Although there is an overall good agreement between the CFD simulation and the CHT simulation, some differences need to be pointed out. The CFD simulation has been made on the stator and the rotor that means that an unsteady method has been used to take into account the phase-lag of the rotor blade passing, as mentioned by Salvadori et al [13]. For the CHT simulation, a radial equilibrium is imposed and the rotor is not considered, which explain the differences on the isentropic Mach at the trailing edge of the stator blade. The aerodynamic behavior is not affected by the coupling process. In fact, as it was previously reported, the inlet non-uniformities does not affect the isentropic Mach [14], but aerodynamic properties has to be in good agreement with experimental data to capture the right fluxes at the interface.

Also a plot of the Nusselt number at $50 \%$ span is presented. The CHT simulation captures the variation of the Nusselt number at the blade interface. A better overall agreement between the CHT simulation and the experimental data is obtained by using a coupling process. On the pressure side, the CHT succeed in capturing the right variation of the Nusselt number. On the suction side, there is a difference between CFD simulation and the CHT simulation. While no variation is captured by the CFD, the CHT simulation succeeds in having a better agreement with experimental data, especially near the leading edge. The differences between experimental data and CHT simulation on the suction side near the trailing edge might be due to the fully turbulent assumption made whereas the experimental data shows a laminar region on the frontal part [15].
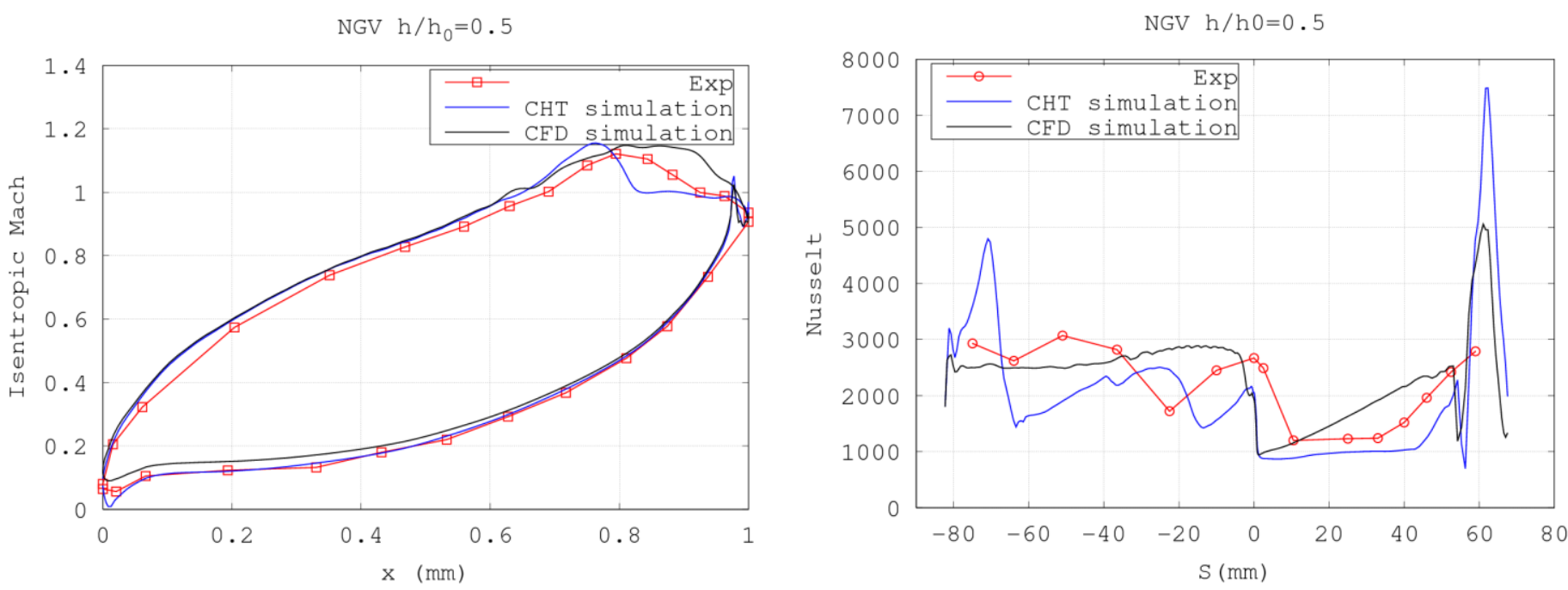

Fig. 3: Isentropic Mach number (left) and Nusselt number (right) comparison at 50\% span.

\subsection{Flat Plate with Thermal Barrier Coating}

The second configuration of interest for HP turbine blade is to take into account the TBC. For this test case shown in Fig.4, a flat plate with $\mathrm{TBC}$ is considered to validate the optimal coefficient approach at high Biot numbers, presented in Tab. 1. Being really thin, the TBC needs to be well discretized. The coupling procedure used does not require the transient thermal solid solution. Since only the steady state is relevant, a second order ordinary equation is solved $(\Delta T=0)$ which allows saving CPU time and stabilizing the coupling process. 


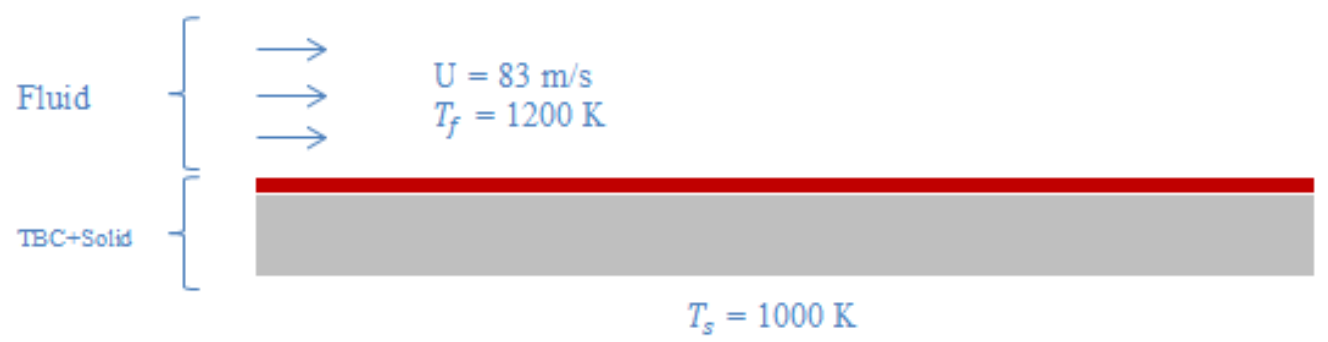

Fig. 4: Thermal barrier coating on flat plate

\section{Test Case Parameters}

The TBC has a thermal conductivity of $1 \mathrm{~W} / \mathrm{m} / \mathrm{K}$ and is further reduced until reaching $0.001 \mathrm{~W} / \mathrm{m} / \mathrm{K}$ in order to have a strong interaction between the fluid and the solid. Therefore, the Biot number, defined by the conductance ratio of both the fluid and the solid, gets very high (Tab. 1).

The fluid used for all simulations is air with the Sutherland's approximations on the temperature and viscosity. The thermal conductivity of the fluid is $\lambda_{f}=0.06 \mathrm{~W} / \mathrm{m} / \mathrm{K}$. The solid thermal conductivity, under the TBC, is always $\lambda_{s}=20$ $\mathrm{W} / \mathrm{m} / \mathrm{K}$, and a fixed temperature of $1000 \mathrm{~K}$ is set at the bottom of the solid.

The accuracy of the results depends mainly on the solver used to compute each domain and the accuracy of the interpolations. The main focus, in this paper, will be to validate this method at the interface by comparing the results with those obtained from a computation without relaxation coefficient at low Biot number.

\section{Results}

In Tab. 1, different thermal conductivities of the TBC, $\lambda_{T B C}$, are tested with both Dirichlet-Robin and NeumannDirichlet conditions. The simulation is considered converged if the infinity norm, $L_{\infty}$, on the temperature at the interface is less than $10^{-3}$. For low Biot number, the Dirichlet-Robin condition is more efficient and converges quickly, but the interaction between the fluid and the solid is not strong enough to allow the Neumann-Dirichlet condition to converge. Moreover, it can be noticed that stronger the interaction gets, faster is the convergence of the Neumann-Dirichlet condition. Indeed the Neumann-Robin condition is known to perform better at high Biot number, since solid thermal gradients are predominant, the normal heat fluxes at the interface is more relevant in the exchange.

This is not the case for the Dirichlet-Robin condition. Dirichlet-Robin condition performs better at low Biot number, however the local optimal parameter allow to strongly stabilizing the simulation. Fields exchanged are then better estimated and the simulation converges faster than the other condition.

Table 1: Convergence of two different boundary treatments.

\begin{tabular}{|c|c|c|c|c|}
\hline$\lambda_{S}[\mathrm{~W} / \mathrm{m} / \mathrm{K}]$ & $\lambda_{T B C}[\mathrm{~W} / \mathrm{m} / \mathrm{K}]$ & \multirow{2}{*}{$B i=\frac{K_{f}}{K_{S+T B C}}$} & \multicolumn{2}{|c|}{ Iteration to convergence $\left(\Delta T=10^{-3}\right)$} \\
\cline { 5 - 5 } \cline { 5 - 5 } & & & Dirichlet - Robin & Neumann - Dirichlet \\
\hline 20 & 1 & 13.56 & 780 & $\mathrm{x}$ \\
\hline 20 & $10^{-1}$ & 112.59 & 880 & 3390 \\
\hline 20 & $10^{-2}$ & 1106.47 & 920 & 1670 \\
\hline 20 & $10^{-3}$ & & 1240 & 1370 \\
\hline
\end{tabular}



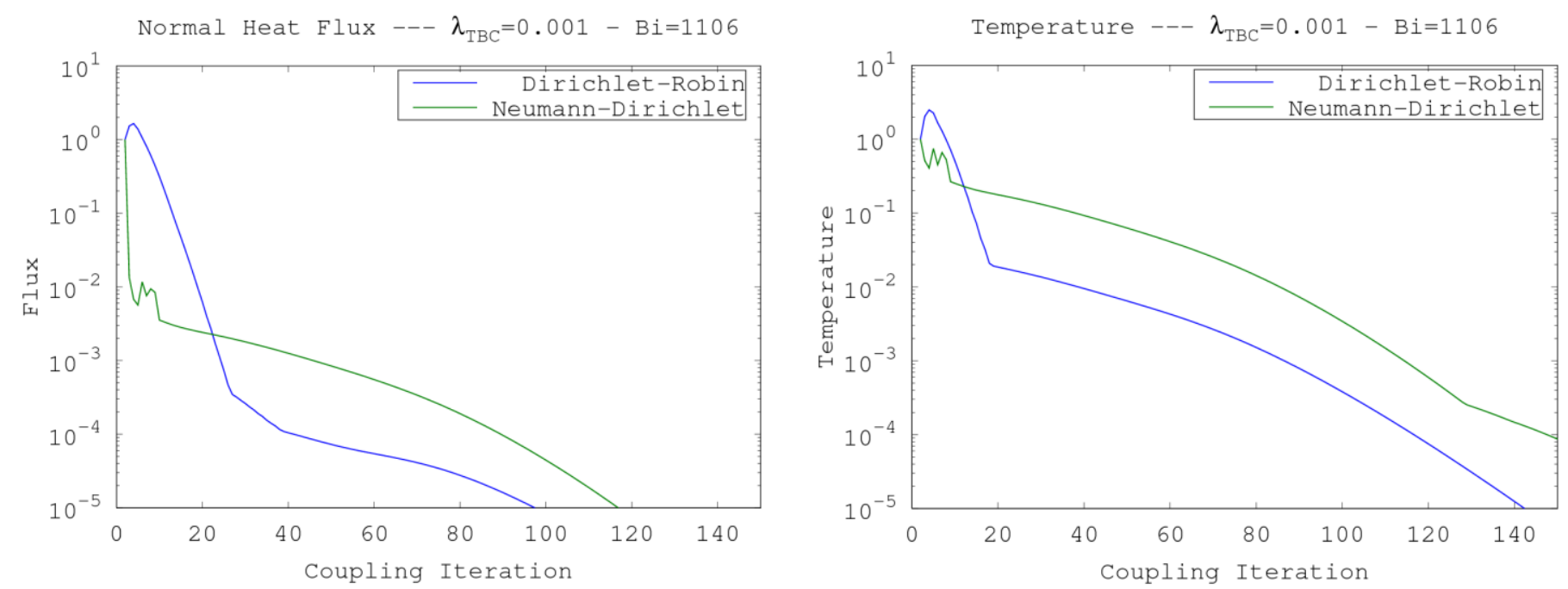

Fig. 5: Convergence $\left(L_{\infty}\right)$ of the normal heat flux (left) and the temperature (right) for $\lambda_{T B C}=0.001 \mathrm{~W} / \mathrm{m} / \mathrm{K}$ and $B i=1106$

The convergence speed is shown in Fig. 5. As it can be noticed, both of the conditions converge for a very low TBC thermal conductivity $\left(10^{-3} \mathrm{~W} / \mathrm{m} / \mathrm{K}\right)$. It is also interesting to note that the Neumann-Dirichlet condition seems to converge faster in the first couple iterations, despite the oscillation. Those oscillations cause a lot of numerical instabilities and point out the complexity of the CHT procedure to converge. The Dirichlet-Robin condition converges without oscillation which contributes to stabilize the simulation.

\section{Conclusion}

Two different applications of interest in HP turbine design have been studied and discussed. The key element of this thermal analysis is the local Biot number that determines whether the thermal interaction is strong or not. In a real engine environment, a variety of situation occurs from the hot streak to the cooling techniques used to protect HP blades which complexify the thermal analysis. The Dirichlet-Robin condition used at the interface boundary shows good agreement with both experimental data and CFD only simulation. Since, the coefficient transferred is locally defined and a better approximation of the normal heat fluxes is made and the coupling process is more stable. That procedure can also be applied with very high Biot number which occurs, for instance, when there is a coupling between a fluid and TBC.

With the development of complex internal cooling system, the simplicity and the accuracy of this method can easily be involved in the design process of HP turbine blade for better thermal analysis. Moreover, the implementation of the rotor in the CHT simulation will help to have a closer comparison with the previous study conducted on the HP turbine configuration and studying the transitional effect on the suction side of the NGV might have a positive impact on the Nusselt number. It will also be interesting to study the effect of the TBC with a CHT simulation on the HP turbine blade using the same methodology as described in this paper.

\section{Acknowledgment}

The authors would like to thank L. Castillon from the French Aerospace Lab and Dr. S. Salvadori from Florence University for letting us uses the meshes and data collected during the European project TATEF-2. Also the authors acknowledge the useful help of J. Mayeur, Dr. J.D. Garaud and R. Moretti.

\section{References}

[1] R.S. Bunker, "Gas turbine heat transfer: ten remaining hot gas path challenges," J. of Turbomachinery, vol. 129, no 2, pp. 193-201, 2007. 
[2] M. P. Bacos, J. M. Dorvaux, S. Landais, O. Lavigne, R Mévrel, M. Poulain, C. Rio, M.-H. Vidal-Sétif, "10 Yearsactivities at Onera on advanced thermal barrier coatings," J. AerospaceLab, no 3, pp. 1-14, 2011.

[3] M. P. Errera, S. Chemin, "Optimal solutions of numerical interface conditions in fluid-structure thermal analysis," $J$. Comput. Phys., vol. 245, pp. 431-455, 2013.

[4] M. P. Errera, G. Turpin, "Temporal multiscale strategies for conjugate heat transfer problems," J. Coupled Syst. Multiscale Dyn., vol. 1, no. 1, pp. 89-98, 2013.

[5] R. El Khoury, M. P. Errera, K. El Khoury, M. Nemer, "Efficiency of coupling schemes for the treatment of steady state fluid-structure thermal interactions," Int. J. Therm. Sci., vol. 115, pp. 225-235, 2017.

[6] M. P. Errera, F. Duchaine, "Comparative study of coupling coefficients in Dirichlet-Robin procedure for fluidstructure aerothermal simulations," J. Comput. Phys., vol. 312, pp. 218-234, 2016.

[7] L. Cambier, J. P. Veuillot, "Status of the elsa software for flow simulation and multi-disciplinary applications," 46th AIAA Aerospace Sciences Meeting and Exhibit, p. 664, 2008.

[8] D. Missoum-Benziane, V. Chiaruttini, J. D. Garaud, F. Feyel, R. Foerch, N. Osipov, S. Quilici, J. Rannou, A. Roos, D. Ryckelynck "Z-set/ZeBuLoN: une suite logicielle pour la mécanique des matériaux et le calcul de structures," in 10e colloque national en calcul des structures, p. 8, 2011.

[9] A. Refloch, B. Courbet, A. Murrone, P. Villedieu, C. Laurent, P. Gilbank, J. Troyes, L. Tessé, G. Chaineray, J. B. Dargaud, E. Quémerais, F. Vuillot, “CEDRE software,” J. AerospaceLab, no 2, pp. 1-10, 2011.

[10] C. A. Felippa, K. C. Park, "Staggered transient analysis procedures for coupled dynamic systems: formulation," Comput. Methods Appl. Mech. Eng., vol. 24, no. 1, pp. 61-111, 1980.

[11] S. Piperno, C. Farhat, B. Larrouturou, "Partitioned procedures for the transient solution of coupled aroelastic problems Part I: Model problem, theory and two-dimensional application," Comput. Methods Appl. Mech. Eng., vol. 124, no. 1-2, pp. 79-112, 1995.

[12] F. Montomoli, P. Adami, S. Della Gatta, "Conjugate heat transfer modelling in film cooled blades," in: ASME Turbo Expo 2004: Power for Land, Sea, and Air. American Society of Mechanical Engineers, pp. 147-156, 2004.

[13] S. Salvadori, F. Montomoli, F. Martelli, P. Adami, K. S. Chana, L. Castillon, "Aerothermal study of the unsteady flow field in a transonic gas turbine with inlet temperature distortions," J. of Turbomachinery, vol. 133, no 3, p. 031030, 2011.

[14] K. L. Gundy-Burlet J. D. Dorney, "Influence of 3D Hot Streaks on Turbine Heat Transfer," International Journal of Turbo and Jet Engines, vol. 14, no 3, p. 123-132, 1997.

[15] B. Khanal, L. He, J. Northall, et al, "Analysis of radial migration of hot-streak in swirling flow through highpressure turbine stage,” Journal of Turbomachinery, vol. 135, no 4, p. 041005, 2013. 